\title{
Survey on Classification, Detection and Prevention of Network Attacks using Rule based Approach
}

\author{
Wrushal K. Kirnapure \\ Yeshwantrao Chavan \\ College Of Engineering, Nagpur
}

\author{
Arvind R. Bhagat Patil \\ Yeshwantrao Chavan \\ College Of Engineering, Nagpur
}

\begin{abstract}
Intrusion detection systems(IDS) has assumes an important part to protect the qualities of PC mostly into two classifications: malignant and irrelevant exercises. Intrusion detection can be accomplish by Categorization. Another machine learning based algorithm for order of information is actualized to network intrusion detection is presented in this paper. The most basic employment is to separate exercises of network are as ordinary or irrelevant while decreasing the misclassification. The goal of Intrusion detection framework (IDS) are to apply all the accessible data keeping in mind the end goal to distinguish the attacks by outcast programmers and abuse of insiders. For Network intrusion detection there are diverse arrangement models have been produced, the most regularly connected strategies are Support Vector Machine(SVM) and Ant Colony both consider their qualities and shortcomings independently. To diminishes the shortcoming, blend of the SVM technique with Ant Colony to take the advantages ofboth. A standard benchmark of information set KDD99 is assessed and actualized as another algorithm. Despite the fact that to increment both the grouping rate and runtime adequacy it is important to actualize the Combining Support Vectors with Ant Colony which beat SVM alone. An individual continuous network dataset and a notable dataset i.e. KDD99 CUP has been actualized as proposed framework. All attack sorts, detection rate, detection speed, false alert rate can be measured by execution of intrusion detection framework IDS.
\end{abstract}

\section{Keywords}

Network,SVM, Ant Colony, KDDCUP 99, Dataset.

\section{INTRODUCTION}

Information technology of today's era have turned out to be extremely testing and also urgent for taking care of the grouping and bunching of huge scale information. For continuous application in PC system, where distinctive sort of intense apparatuses are utilized to secure the processing assets, for example, exponential increment in-size and extensive scale information inputs. In this paper ,intrusion detection framework term actualize as a reacting procedure to distinguish and deal with the pernicious exercises focused by networking assets and computational assets. An intrusion detection framework IDS finds to uncover or un cover design or qualities that could prompt to irrelevant or anomalous exercises. An intrusion detection framework is detailing of equipment and programming elements, this mix executed to look unforeseen occasions in every one of the three tenses; that demonstrates an attack will happen, is happening or has as of now happened.

\section{RELATED WORK}

The[1] idea of multiscale is presented. We utilize diagrams of various scales built by separating the video outlines into squares of various sizes to accomplish more human eye versatility. At that point the spatial elements, in particular luminance, chrominance and surface, are separated specifically from discrete cosine change coefficients while the transient data are removed from the movement vectors to frame the heuristic lattices. Next, the heuristic grids are utilized as a feature of the ant colony enhancement handle. Every heuristic lattice is utilized to direct the ants in the calculation and the ants store pheromone on the diagram. The pheromone is redesigned through constriction and vanishing in this manner framing spatial/fleeting saliency maps. At last, the spatial and fleeting saliency maps of every scale are melded through adaptive 2 combination, and maps of various scales are intertwined through direct combination. Since the model is developed utilizing data in packed area independently, the decompression procedure is stayed away from to spare additional time and to be appropriate for recordings transmitted on the system. Plus, the proposed technique has been widely tried on a few video databases with successions in different scenes. Through investigations it can be seen that in both quantitative assessment scores and natural visual impacts, the calculation in this paper displays a superior performance contrasted with the differentiation techniques in this paper. In[2] this paper, we introduce a novel calculation, ACCMLF, which joins ant colony bunching with multilevel framework to diminish the runtime in the vast scale PPI systems. In the first place, utilize another coordinating strategy to coarsen the first huge scale PPI arrange, and get a littler PPI organize. At that point, utilize the ant colony bunching calculation to group the got arrange. At last, get the bunching consequence of unique system through decoarsening and utilize the refinement to maintain a strategic distance from the come about because of falling into the neighborhood ideal. Analyzes in some expansive scale systems demonstrate that the recognizing rate of ACC-MLF has significantly enhanced rather than ACC-FMD, and ACCMLF can improve grouping brings about some assessment measurements while contrasted and ACC-FMD, MCODE, MINE and Core calculations. redone and proficient system intrusion detection frameworks utilizing delicate figuring to expand general system security through particular system security. This paper[4] presents the Mobile Network Defense (MND), a lightweight intrusion detection framework. MND is organically displayed on the conduct of a populace of ants, giving it many advantages over conventional safety efforts. Every ant in the virtual colony can recognize one specic metric of the present condition of a PC. In blend, the aftereffects of these basic tests can indicate specic attacks, while the dynamic way of the MND offers performance benets over the customary static setup. This paper will demonstrate how the organically displayed MND offers a $34 \%$ change in detection time over other operator based frameworks, and gives more effective intrusion detection stage than a static model as for CPU use, making the framework alluring for use crosswise over many sorts of cell phones. The system[5] gets and forms dim level pictures 
through one or different camera units observing certain area(s) by means of a neighborhood (LAN) and is fit for consolidating data from various camera units to get an accord choice. It can be prepared to recognize certain sort of intrusions, for instance people on foot, a gathering of walkers, vehicles, pets, and so on., and limits false cautions because of other non-intrigued intrusions. As a contextual analysis, we intend to distinguish person on foot/vehicle in a perception territory. Our vision-based intrusion detection approach comprises of two primary strides: foundation subtraction based theory era (HG) and appearance based speculation check (HV). HG speculates conceivable dangers (intrusions), and HV checks those theories utilizing a Gabor channel for highlight extraction and support vector machines (SVMs) for characterization. The framework has been tried in an unconstrained open air environment, delineating great performance. This[3] paper introduces a stage forward in this bearing where the IDS show addresses a particular part of the system attacks normally identified at port 7 in UDP. Port sweeps in UDP represent a sizable bit of the Internet movement and nearly little research describes security in UDP port output action. To meet the developing pattern of attacks and other security challenges in the constantly advancing web field, this is paper displays a computationally clever intrusion detection instrument utilizing swarm insight worldview, especially ant colony improvement, to break down specimen arrange follows in UDP The fundamental point of this study[6] is introducing port outputs. This work goes for producing a DOS assault intrusion detection framework by 3 utilizing support vector relapse and enhancing this calculation by consolidating two calculations of ant colony and firefly. The firefly calculation changes ants' positions by playing out a lo cal pursuit and firefly performance would be upgraded by diminishing arbitrary parameters. Standard KDD Cup 99 arrangement has been utilized for the assessment of intrusion detection framework. This arrangement incorporates 41 properties among which 9 properties have been chosen. The displayed framework has 99.57 This[7] prompted to an expanding requirement for proficient techniques for perceiving intrusions keeping in mind the end goal to secure the frameworks. Existing models of intrusion detection frameworks (IDS) have created significant performance however regularly has the powerlessness for recognizing multilevel classes of attacks combined with high preparing time for classifiers. These downsides prompted to half breed models that consolidate the different qualities of single classifiers in the meantime evading their shortcomings for better performance. In this paper, a correlation of such half and half models is completed. The goal is to decide their performances and disconnect their shortcomings. In this manner, an exploration hole is built up for more productive intrusion detection models. $\operatorname{In}[8]$ this paper, we outline a conveyed grouping framework without requiring any earlier model data. In particular, at every nearby sensor, different double support vector machine (SVM) based classifiers are utilized and every classifier is prepared to recognize one class from the rest. At the combination focus, the Dempster-Shafer hypothesis is embraced to adequately consolidate the confirmation from all SVMs with suitably characterized essential likelihood assignments. A ultimate conclusion is made by selecting the class with the most elevated conviction. Hypothetical performance expectation strategies are proposed for the planned arrangement framework. Through tests on a manufactured dataset and the benchmark 1999 KDD intrusion detection dataset, we demonstrate the viability of the assessment strategy and the prevalence of the proposed framework over the traditional Bayesian cost based combination manage in this specific circumstance. $\operatorname{In}[9]$ this theory, memory standard and ant colony calculation are melded and connected in intrusion detection framework. The technique for controlling pheromone utilized as a part of ant colony calculation is connected to reenact the memory procedure of human cerebrum, and the idea of pheromone is advanced. The procedures of remembering and overlooking are sensibly translated through the expansion and diminishing of pheromone, and in the calculation, new memory calculation is shaped to complete the procedures of retaining and overlooking in the wake of considering the effect of anomalous qualities weight an incentive on pheromone. Cases demonstrate that the calculation is fit for acknowledging remembering and overlooking procedures and expanding the heartiness and self versatility of IDS. This paper[10] proposes a novel intrusion detection approach by applying ant colony improvement for highlight choice and SVM for detection. The intrusion components are spoken to as chart ere hubs, with the edges between them meaning the including of the following element. Ants cross through the chart to include hubs until the halting standard is fulfilled. The fisher separation rate is embraced as the heuristic data for ants' traversal. So as to abstain from preparing of countless classifier, the minimum square based SVM estimation is embraced. At first, the SVM is prepared in light of lattice hunt technique to get separation work utilizing the preparation information in view of all components accessible. In[11] this paper, we exhibit a Genetic Algorithm (GA) approach with an enhanced starting populace and determination administrator, to proficiently identify different sorts of system intrusions. GA is utilized to enhance the inquiry of assault situations in review records, because of its great adjust investigation/misuse; it gives the subset of potential attacks which are available in the review document in a sensible handling time. In the testing stage the Network Security Laboratory-Knowledge Discovery and Data Mining (NSL-KDD99) benchmark dataset has been utilized to recognize the 4 abuse exercises. By consolidating the IDS with Genetic calculation builds the performance of the detection rate of the Network Intrusion Detection Model and diminishes the false positive rate. The performance of GAAIS is assessed for identifying a few sorts of steering attacks mimicked utilizing the NS2 test system, for example, Flooding, Blackhole, Neighbor, Rushing, and Wormhole. Trial comes about demonstrate that GAAIS is more effective in examination with $\operatorname{In}[12]$ this paper, we present an Intrusion comparable methodologies. Detection framework (IDS) based Hybrid Evolutionary Neural Network (HENN). This[13] paper displays a fluffy hereditary quicker as the pursuit space of the subsequent administer way to deal with recognizing system intrusion. set is much minimized when contrasted with the Paper displays the aftereffects of the proposed unique informational collection. This makes IDS speedier and framework as far as precision, execution time, keen. We generate conceivable intrusions and memory allotment. To execute and which frames the reason for recognizing intrusions measure the performance of the framework the on the system activity. Our technique displays a KDD99 benchmark dataset is utilized. The KDD99 high detection rate with low false positives. We dataset is a benchmark dataset that scientists have utilized DARPA Dataset for introductory preparing use in different system security investigates. furthermore, testing reason. Hereditary calculation incorporates an advancement and gathering that uses a chromosome like information This paper[17] presents and researches structure and build up the chromosomes utilizing the conduct of dynamiCS, an element clonal choice, hybrid and transformation administrators. determination calculation, 
intended to have such Fuzzy govern sorts organize assault information. properties of self-adjustment. The impacts of three important framework parameters: tolerisation $\operatorname{In}[14]$ this paper, we proposed an approach period, enactment limit, and life expectancy depend on hereditary calculation (GA) and manufactured investigated. The capacities of dynamiCS to perform resistant framework (AIS), called GAAIS, for incremental learning on met information, and element intrusion detection in AODV-based to adjust to novel information are additionally demonstrated. MANETs. GAAIS can adjusting to network topology changes utilizing two This paper[18] proposes a detection demonstrate, redesigning strategies: halfway and add up to. Every ant framework with support vector machine, ordinary element vector separated from system which utilizes ant framework, a variety of activity is spoken to by a hypersphere with settle ant colony improvement, to sift through the range. An arrangement of circular finder is generated redundant and irrelevant components for support utilizing NicheMGA calculation for covering the vector machine grouping calculation. nonself space. Round identifiers are utilized KDD99, which is a benchmark dataset utilized for recognizing oddity in system activity. for abnormality detection, has been embraced here. 5 Each occasion in KDD99 has been spoken to by 41 highlights which additionally has some redundant or irrelevant components. Ant framework has been utilized to expel those redundant and irrelevant elements. The chose highlight subset utilizing ant framework is then approved utilizing support vector machine. The exploratory outcomes demonstrated that the performance of the order calculation, when prepared with the diminished list of capabilities, has been progressed. The performance measures utilized as a part of this examination are genuine positive rate, false positive rate, and accuracy. movement performed by the clients and if any kind of unfortunate behavior action is recognized then it caution to the manager to turn away it. Here, the practice is done on KNN classifier with ACO (ant colony advancement) strategy to uncover the gatecrashers and make an utilization of KDDCUP dataset to classify distinctive class of assault. The reenactment examination of proposed framework is done utilizing exactness and false alert rate (FAR) performance parameter. Our proposed framework generates more accurate outcomes than the current technique. $\operatorname{In}[19]$ this paper we have proposed a transformative calculation to enlist fluffy characterization rules. The calculation utilizes an ant colony streamlining based nearby searcher to enhance the nature of last fluffy grouping framework. The proposed calculation is performed on intrusion detection as a high-dimensional grouping issue. Comes about demonstrate that the actualized transformative ACO-Based calculation is equipped for delivering a dependable fluffy govern based classifier for intrusion detection. The[22] principle objective of assault detection framework is order of framework exercises into two fundamental gatherings of ordinary exercises and intrusion exercises. However many reviews have been done in the field of intrusion detection, finding a strategy with min mistake and max precision is still a test. The primary point of this review is displaying a DOS assault intrusion detection framework by utilizing support vector relapse and advancing this calculation by consolidating two calculations of ant colony and firefly. The firefly calculation alters ants' positions by playing out a nearby pursuit and firefly performance would be streamlined by diminishing irregular parameters. Standard KDD Cup 99 arrangement has been utilized for the assessment of intrusion detection framework. This arrangement incorporates 41 properties among which 9 properties have been chosen. The displayed framework has $99.57 \%$ precision rate and $0.0064 \%$ mistake detection rate. The[20] nearness of intrusion assault follows in system movement design is by all accounts major undermining to digital group. Amid 10 years, numerous preventive and detection measures have had been created to beat these illegal exercises however the development of zeroday misuses which has basic conduct as intrusion follows discover hard to determine the commentators nearness in system activity designs. Alternate pundits confronted by preventive and detection measures are lion's share of intrusion follows takes after as would be expected conduct in system movement design investigation. Contemporary preventive or criminologist measures have been advanced either as neither one-hand approach nor half and half methodologies. Target of this paper is to elaborate talk about the detection and preventive measures developed still and their blemishes acquired in their methodologies. Along with[23] the expansion of system attacks, arrange data security has turned into an all around concerned issue. At present, standard intrusion detection frameworks have the all inclusive issues of enormous caution data and high false alert rate. Accordingly, an information mining innovation is proposed in this article so as to diminish the quantity of the false alerts generated by intrusion detection frameworks and in the mean time enhance the detection precision, wherein such information mining innovation is an unsupervised $\operatorname{In}[21]$ this work, intrusion detection utilizing grouping technique in light of half breed ant colony information mining calculation is examined. The calculation and can be utilized to distinguish gatecrashers' intrusion detection framework manages the aggregate practices, without the need to know6 the earlier information. Then, we receive $\mathrm{K}$-implies bunching calculation to accelerate the merging rate of the Ant Colony calculation. Really, the test result demonstrates that the technique proposed accordingly has higher detection rate however bring down false caution rate. Classification[24] utilizing ant colony advancement (ACO) calculation gives a decent strategy to clients to comprehend the information got from occasion log documents, which can additionally help in building a framework profile and figuring out if intrusions have occurred in the framework. To assess the obstruction shirking strategy, the parameters utilized are along the lines of effortlessness of guidelines shaped, number of terms present in the tenets and furthermore the prescient exactness of the test information on the preparation set utilizing the standards got. We have attempted to dissect changes in the manage arrangement handle for various limits, and for various circumstances inside the way toward producing rules. We appear through our assessment that the hindrance evasion strategy to ACO performs superior to the prevalent ant-mineworker calculation by building straightforward tenets with an enhanced prescient exactness. to as "zero" day attacks, yet have high false positive rates. False positive occasions happen when an action is hailed for examination yet it was resolved to be amiable upon investigation. Computational power and significant assets are squandered when the irrelevant information is handled, information hailed, expert cautioned, and the irrelevant information is at long last neglected. With an end goal to make intrusion detection frameworks more proficient the false positive rate must be lessened. This paper proposes a model for decreasing false positives utilizing information mining techniques by joining support vector machines (SVM), choice trees, and Naive Bayes.

These[27] techniques upgrading the detection rate of the intrusions which is extremely viable. Discriminant capacity is extremely basic in isolating the typical and inconsistency conduct accurately. The support vector machine based 
arrangement calculation is utilized to order the intrusions accurately by utilizing the discriminant work. The compelling discriminant capacity will be accurately distinguishes the information into intrusion and oddity. The assessment of the discriminant is important in the assessment of the intrusion detection framework. Performance The[25] fundamental idea of the technique is to of intrusion detection framework relies on upon the create the bunch by swarm insight decision of the discriminant work. based grouping. With the arranged information cases, oddity information bunches can be effectively Intrusion Detection System (IDS)[28] is recognized by ordinary group proportion. And afterward used to protect the information honesty and the distinguished bunch can be utilized as a part of genuine information privacy from attacks. With a specific end goal to detection. In the conventional grouping based recognize the kind of assault in IDS, diverse intrusion detection calculations, bunching approachs like different information mining utilizing a straightforward separation based metric and techniques exist. Be that as it may, some are exceptionally time detection in view of the focuses of bunches, expending and relentless. In this manner we have which for the most part corrupt detection exactness proposed the use of SVM (Support Vector and proficiency. Our approach in light of Machine) for arrangement of assault from vast swarm knowledge can settle these issues measure of crude intrusion detection datasets successfully. The trial result demonstrates that on standard PCs. SVM is an our approach can distinguish obscure intrusions technique which is utilized as a part of information mining to extricate productively in the genuine system associations. anticipated information. We have utilize KDDCUP'99 IDS database for order. Intrusion detection systems[26] screen system or host bundles trying to $\operatorname{In}[29]$ this paper, we demonstrate the recognize noxious exercises on a framework. utilization of managed segment participation Anomaly detection frameworks have achievement in preprocessing strategy to distinguish vague uncovering new attacks, generally alluded bundles. We propose an integrated model that 7 brings about enhanced characterization precision by unequivocally bunching vague bundles to conquer its misclassification. The oddity of our approach lies being used of non-fresh grouping techniques like fluffy c-implies (FCM) and unpleasant k-implies (RKM) that can display vagueness. Encourage, we likewise analyzed whether FCM bunching and RKM grouping can decide class of uncertain parcels precisely or around. The support vector machine (SVM) and J48 classifiers comes about got on two standard informational indexes are exhibited and analyzed. In[30] data insurance, Intrusion Detection System (IDS) is utilized to defend the information secrecy, uprightness and framework accessibility from different sorts of attacks. Information mining is a proficient guile connected to intrusion detection to discover another framework from the gigantic system information and additionally it used to lessen the strain of the manual aggregations of the typical and anomalous conduct designs. This bit of composing audits the current situation with information mining techniques and analyzes different information mining techniques used to execute an intrusion detection framework, for example, Support Vector Machine, Genetic Algorithm, Neural system, Fuzzy Logic, Bayesian Classifier, K-Nearest Neighbor and choice tree Algorithms by highlighting an advantage and disadvantages of each of the techniques. In[31] this paper, an exertion has been made to propose a productive intrusion detection display by mixing able information mining techniques, for example, Fuzzy-Cimplies bunching, Artificial neural network(ANN) and support vector machine (SVM), which is significantly extemporizes the expectation of system intrusions. We actualized the proposed IDS in MATLAB adaptation R2013a on a Windows PC having $3.20 \mathrm{GHz}$ CPU and 4GB RAM. The examinations and assessments of proposed strategy were performed with Corrected KDD glass 99 intrusion detection dataset and we utilized affectability, specificity and exactness as the assessment measurements. We accomplished detection exactness of around $99.66 \%$ for DOS attacks, $98.55 \%$ for PROBE, $98.99 \%$ for R2L and $98.81 \%$ for U2R attacks. Results are contrasted and relevant existing techniques in order to demonstrate productivity of our model.

\section{PROPOSED SYSTEM}

Intrusion Detection Systems (IDSs) are designed to defend computer systems from various cyber attacks and computer viruses. Intrusion Detection Systems (IDSs) are designed to defend computer systems from various cyber attacks and computer viruses. IDSs build effective classification models or patterns to distinguish normal behaviors from abnormal behaviors that are represented by network data. To classify network activities (in the network log) as normal or abnormal while minimizing misclassification .To defend computer systems from various cyber attacks and computer viruses. To balance the performance of IDS in terms of efficiency and accuracy.

Intrusion Detection Systems (IDSs) are designed to defend computer systems from various cyber attacks and computer viruses.

1. IDSs build effective classification models or patterns to distinguish normal behaviors from abnormal behaviors that are represented by network data.

2. To classify network activities (in the network log) as normal or abnormal while minimizing misclassification .To defend computer systems from various cyber attacks and computer viruses.

3. To balance the performance of IDS in terms of efficiency and accuracy.

\section{LIMITATIONS OF EXISTING STRATEGIES}

1) A ultiscale compressed video saliency detection model based on ant colony optimization Theoretical analysis is difficult and more complex to implement.

2) Ant Colony Clustering Approach Combined with Multilevel Framework for Functional Module Detection in Large-Scale PPI Networks Sequences of random decisions (not independent)

3) Intelligent Perpetual Echo Attack Detection on User Datagram Protocol Port 7 Using Ant Colony Optimization Probability distribution changes by iteration.

4) A Biologically Modeled Intrusion Detection System for Mobile Networks Research is experimental rather than theoretical so risky to develope such system.

5) A distributed visual surveillance system 5.Time to convergence uncertain (but convergence is guaranteed

6) DOS intrusion attack detection by using of improved SVR Due to less no of parameters the ant colony method takes more time to process the input dataset and hence for detecting the intrusion make less efficient.

7) Analysis and evaluation of hybrid intrusion detection 
system models For detecting and improving the performance of the system they had used the hybrid model which is is not efficient for solving the huge dataset file.

8) Data-based distributed classification and its performance analysis The preprocessed model information is not available hence if the attack is generated and detected it is not saved in advance for further enhancement in the system to save time for detecting future attacks of similar type.

9) Intrusion detection system based on ant colony memory principle The concept pheromone is used in this system which is not memory efficient and then as author has used the ant colonymethod, that why requires more amount of the memory to process huge amount of data.

10) Ant colony optimization based network intrusion feature selection and detection Ants traverse through the graph to add nodes until the stopping criterion is satisfied. If there is an the condition like new attack is not recognisable then system undergoes in the deadlock situation.

11) Intrusion detection system using genetic algorithm As initial population is not fixed the system may process small dataset file for the long time if initial population is given to be small.

12) Intrusion Detection System Using Genetic Algorithm Input features, network structure and connection weights should be known in advance to work that algorithm but in practacly it is hard to detect the network structure to user end as it is completely dynamic in nature.

13) Intrusion detection system using fuzzy genetic algorithm fuzzy-genetic approach uses the chromosomes using selection, crossover and mutation operators which is costly operations.

14) A hybrid approach for dynamic intrusion detection in ad hoc networks using genetic algorithm and artificial immune system Can be used only in manet or small ring structure topology for detection of Flooding, Blackhole, Neighbor, Rushing.

15) Detectors generation using genetic algorithm for a negative selection inspired anomaly network intrusion detection system Uses deterministic crowding Niching technique which comsumes time to process and detect the attack.

16) IGIDS: Intelligent intrusion detection system using genetic algorithms Can be used only in the pruning best individuals in the rule set database for large network it is not usful.

17) Towards an artificial immune system for network intrusion detection: an investigation of dynamic clonal selectionEvolutionary Computation The abilities of dynamiCS to perform clonnig of the complete data hence not memory efficient solution.

18)SVM for network anomaly detection using ACO feature subset More resources is used to calculate the SVM rules.

19)Induction of Fuzzy Classification Systems Using Evolutionary ACO-Based Algorithms Used only in the high dimensional classification problems.
20) A proposed hybrid framework for improving supervised classifiers detection aecuraev over intrusion trace This system has evolved either as neither one-hand approach nor hybrid approaches.

21) A KNN-ACO approach for intrusion detection using KDDCUP99 dataset2016 3rd International Conference on Computing for Sustainable Global Development It is not an automated system admin needs to check and verify that attack

22) DOS intrusion attack detection by using of improved SVR2015 International Congress on Technology This system keeps network lots busy even if no attack available.

23) Intrusion Detection Alarm Filtering Technology Based on Ant Colony Clustering Algorithms System uses the kmean clustering algorithm which is not safe.

24) Evaluating an Obstacle Avoidance Strategy to Ant Colony Optimization Algorithm for Classification in Event Logs Intrusion pull are created in excess quantity which provide loads in the network.

25) An unsupervised anomaly intrusion detection algorithm based on swarm intelligence Swarm intelligence technique is used which is too complex to understand and modify.

26) Reducing false positives in intrusion detection systems using data-mining techniques utilizing support vector machine False positive events occur when an activity is flagged for investigation yet it was determined to be benign upon analysis. Computational power and valuable resources are wasted when the irrelevant data is processed.

27) Effective discriminant function for intrusion detection using SVM System requires more bandwidth to compute both things simultenuosly.

28) Classification of attacks Using Support Vector Machine (SVM) on KDDCUP99 IDS Database This method also very time consuming and laborious.

29) Handling ambiguous packets in intrusion detection Two standard datasets needs to maintain and hence provide the huge amount of the data processing and tracing the process which are currently running in the system,

30) Efficient classification mechanism for network intrusion detection system based on data mining techniques Support Vector machine, Machine, Genetic Algorithm, Neural network, Fuzzy Logic, Bayesian Classifier, KNearest Neighbor and decision tree Algorithms requires to run simultenuosly hence more load on the system to process that data.

31) Confederation of FCM clustering, ANN and SVM techniques to implement hybrid NIDS using corrected KDD cup 99 dataset System requires huge resources to run flowlessly.

\section{CONCLUSION}

We will work with this examination HIDS additionally NIDS. We found piles of existing work in HIDS basically we endeavor to increase the area rate NIDS with different attack using proposed counts. As future work, we are considering fusing the security protecting OLAP with the proposed system in demand to improve the sufficiency and the versatility of IDS framework. Attacks are classified according to the 
administer generation of SVM. With the assistance of Ant Colony the assault classification and detection rate is expanded.

\section{ACKNOWLEDGMENTS}

For everything I achieved, the credit goes to all those who had really helped us to complete this work successfully. Extremely thankful to Prof. A. R. Bhagat Patil for guidance and review of this paper also thanks the all faculty members.

\section{REFERENCES}

[1] Cuiwei Li,Qin Tu,MaozhengZhao,JunXu,Aidong Men,Amultiscale compressed video saliency detection model based on ant colony optimization, 2015 IEEE/CIC International Conference on Communications in China (ICCC) Year: 2015

[2] Hongxin Liu; JunzhongJi; Cuicui Yang; JiaweiLv; Xiuzhen Zhang,Ant Colony Clustering Approach Combined with Multilevel Framework for Functional Module Detection in Large-Scale PPI Networks , 2014 IEEE/WIC/ACM International Joint Conferences on Web Intelligence (WI) and Intelligent Agent Technologies (IAT)

[3] Abhishek Gupta; Om JeePandey; MahendraShukla; Anjali Dadhich; Anup Ingle; Vishal Ambhore,Intelligent Perpetual Echo Attack Detection on User Datagram Protocol Port 7 Using Ant Colony Optimization, 2014 International Conference on Electronic Systems, Signal Processing and Computing Technologies

[4] Brian C. Williams; Errin W. Fulp, A Biologically Modeled Intrusion Detection System for Mobile Networks , 2010

[5] International Conference on Broadband, Wireless Computing, Communication and Applications Xiaojing Yuan; Zehang Sun; Y. Varol; G. Bebis ,A distributed visual surveillance systemProceedings of the IEEE Conference on Advanced Video and Signal Based Surveillance, 2003.

[6] Zohreh Sadat Hosseini; Seyyed Javad Seyyed Mahdavi Chabok; Seyyed Reza Kamel, DOS intrusion attack detectionby using of improved SVR , 2015 International Congress on Technology, Communication and Knowledge (ICTCK)

[7] FaridLawan Bello; KiranRavulakollu; Amrita,Analysis and evaluation of hybrid intrusion detection system models , 2015 International Conference on Computers, Communications, and Systems (ICCCS .

[8] Forman, G. 2003. An extensive empirical study of feature selection metrics for text classification. J. Mach. Learn. Res. 3 (Mar. 2003), 1289-1305.

[9] Brown, L. D., Hua, H., and Gao, C. 2003. A widget framework for augmented interaction in SCAPE.

[10] Y.T. Yu, M.F. Lau, "A comparison of MC/DC, MUMCUT and several other coverage criteria for logical decisions", Journal of Systems and Software, 2005, in press.

[11] Salah EddineBenaicha; LaliaSaoudi; Salah Eddine Bouhouita Guermeche; OuardaLounis,Intrusion detection system using genetic algorithm2014 Science and Information Conference.
[12] Fan Li, Hybrid Neural Network Intrusion Detection System Using Genetic Algorithm 2010 International Conference on Multimedia Technology.

[13] YogitaDanane; ThaksenParvat, Intrusion detection system using fuzzy genetic algorithm2015 International Conference on Pervasive Computing (ICPC).

[14] FatemehBarani, A hybrid approach for dynamic intrusion detection in ad hoc networks using genetic algorithm and artificial immune system ,2014 Iranian Conference on Intelligent Systems (ICIS).

[15] AmiraSayed A. Aziz; MostafaSalama; AboulellaHas sanien; Sanaa EL-Ola Hanafi , Detectors generation using genetic algorithm for a negative selection inspired anomaly network intrusion detection system , 2012 Federated Conference on Computer Science and Information Systems (FedCSIS)

[16] K G Srinivasa; Saumya Chandra; SiddharthKajaria; Shilpita Mukherjee, IGIDS: Intelligent intrusion detection system using genetic algorithms , 2011 World Congress on Information and Communication Technologies

[17] Jungwon Kim; P. J. Bentley , ”Towards an artificial immune system for network intrusion detection: an investigation of dynamic clonal selectionEvolutionary Computation, 2002. CEC '02.Proceedings of the 2002.

[18] TahirMehmood; Helmi B MdRais , SVM for network anomaly detection using ACO feature subset , 2015 International Symposium on Mathematical Sciences and Computing Research (iSMSC)

[19] Mohammad SanieeAbadeh; JafarHabibi; EmadSoroush, "Induction of Fuzzy Classification Systems Using Evolutionary ACO-Based AlgorithmsFirst Asia International Conference on Modelling Simulation (AMS'07)

[20] VidhyaSathish; P. Sheik Abdul Khader , A proposed hybrid framework for improving supervised classifiersdetection aecuraev over intrusion trace2016 International Conference on Electrical, Electronics, and Optimization Techniques (ICEEOT)

[21] SakchiJaiswal; KhushbooSaxena; Amit Mishra; Shiv K. Sahu , A KNN-ACO approach for intrusion detection using KDDCUP'99 dataset2016 3rd International Conference on Computing for Sustainable Global Development (INDIA Com)

[22] Zohreh Sadat Hosseini; Seyyed Javad Seyyed Mahdavi Chabok; Seyyed Reza Kamel , DOS intrusion attack detection by using of improved SVR2015 International Congress on Technology, Communication and Knowledge (ICTCK)

[23] Xu Yang; Zhao Hui , Intrusion Detection Alarm Filtering Technology Based on Ant Colony Clustering Algorithm2015 Sixth International Conference on Intelligent Systems Design and Engineering Applications (ISDEA)

[24] R. Chandrasekar; R. K. Suresh; S. G. Ponnambalam, "Evaluating an Obstacle Avoidance Strategy to Ant Colony Optimization Algorithm for Classification in Event Logs2006 International Conference on Advanced Computing and Communications 
[25] Yong Feng; Zhong-Fu Wu; Kai-Gui Wu; Zhong-Yang Xiong; Ying Zhou, An unsupervised anomaly intrusion detection algorithm based on swarm intelligence2005 International Conference on Machine Learning and Cybernetics

[26] Kathleen Goeschel, Reducing false positives in intrusion detection systems using data-mining techniques utilizing support vector machines, decision trees, and naive Bayes for off-line analysisSoutheastCon 2016

[27] R. Ravinder Reddy; Y. Ramadevi; K. V. N Sunitha , Effective discriminant function for intrusion detection using SVM2016 International Conference on Advances in Computing, Communications and Informatics (ICACCI)

[28] Manjiri V. Kotpalliwar; RakhiWajgi , "Classification of Attacks Using Support Vector Machine (SVM) on KDDCUP'99 IDS Database2015 Fifth International
Conference on Communication Systems and Network Technologies

[29] TheyaznHassnHadi; Manish R. Joshi , Handling ambiguous packets in intrusion detection2015 3rd International Conference on Signal Processing, Communication and Networking (ICSCN)

[30] A. S Subaira; P. Anitha , "Efficient classification mechanism for network intrusion detection system based on data mining techniques: A survey2014 IEEE 8th International Conference on Intelligent Systems and Control (ISCO)

[31] A. M. Chandrasekhar; K. Raghuveer, Confederation of FCM clustering, ANN and SVM techniques to implement hybrid NIDS using corrected KDD cup 99 dataset2014 International Conference on Communication and Signal Processing 